\title{
Cartilage status in FAl patients - results from the Danish Hip Arthroscopy Registry (DHAR)
}

\author{
Bent Lund ${ }^{1, *}$, Torsten Grønbech Nielsen ${ }^{2}$, and Martin Lind ${ }^{2}$ \\ 1 Department of Orthopaedics, Horsens Regional Hospital, 8700 Horsens, Denmark \\ 2 Department of Orthopaedics, Aarhus University Hospital THG, 8000 Aarhus C, Denmark
}

Received 5 December 2016, Accepted 21 March 2017, Published online 14 June 2017

\begin{abstract}
Introduction: The femoroacetabular impingement (FAI) morphology is associated with specific cartilage lesions, which are suspected to be early stages of the osteoarthritic development, which can be the end result of FAI. The cartilage status of FAI afflicted hip joints at the time of arthroscopic management is not fully elucidated. This study from the Danish Hip Arthroscopy Registry (DHAR) will try to show data on the cartilage status from a large cohort. Data from a national registry potentially represent large amounts of population-based epidemiological information from multiple centres and surgeons. Therefore, outcome data might be more reliable for a specific surgical intervention.

Methods: This study includes patients operated for symptomatic FAI from January 2012 until December 31st 2013, with a minimum of two-year follow-up and being registered in DHAR. The extent of cartilage damage at the time of surgery is reported and the Patient Related Outcome Measures (PROM) outcome data are presented.

Results: Data from a total of 686 FAI procedures in 1082 patients from January 2012 until December 31st 2013 were extracted from DHAR. Cartilage injuries were found in $88 \%$ of cases, mainly on the acetabular side. Overall PROM including pain scores improved significantly from preoperative status to follow-up one and two years postoperatively. The Copenhagen Hip and Groin Outcome Score (HAGOS), Hip Sports Activity Scale (HSAS) and global hip function showed less improvements in patients with more severe acetabular cartilage injury.

Discussion: The majority of patients with femoroacetabular impingement (FAI) undergoing hip arthroscopy have significant cartilage changes at the time of surgery primarily at the acetabulum and to a lesser degree at the femoral head. During FAI surgery the majority of patients have cartilage debridement performed but rarely cartilage repair. The presence of severe cartilage injury at the time of arthroscopic FAI surgery results in reduced subjective outcome and hip function.
\end{abstract}

Key words: FAI, Cartilage, HAGOS, National Registry.

\section{Introduction}

Several studies have demonstrated evidence supporting the theory that FAI is a strong risk factor for the development of secondary osteoarthritis of the hip [1-6]. FAI morphology is associated with specific cartilage lesions, which are suspected to be early stages of the osteoarthritic development. The cartilage status of FAI afflicted hip joints at the time of arthroscopic management is not fully elucidated. Similarly there is still limited knowledge on the impact of cartilage injury on outcome after arthroscopic management of FAI. A systematic review from 2013 by Hetaimish et al. states that although FAI can be managed successfully by arthroscopic techniques, the current knowledge of the clinical outcome after FAI

\footnotetext{
*Corresponding author: bentlund@rm.dk
}

surgery is reported with a generally low evidence level mainly as case studies and with a lack of consistency in outcome reporting [7]. Cartilage pathology in FAI is believed to be caused by the impaction of both acetabular and femoral head cartilage edges onto the cam and pincer deformities of the FAI affected hip joint. The cartilage injuries in FAI are characterized by specific changes [8]. Early cartilage changes present as a bulging cartilage surface at the chondrolabral junction (wave sign), further lesion development results in a full thickness delamination and flap formation. Later changes are full thickness defects in the affected areas [9].

Presently several surgical strategies exist for the management of FAI-related cartilage lesions. These range from debridement of bulging and loose cartilage flaps, suturing and gluing of cartilage flaps to different repair techniques for areas of cartilage loss [10-12]. The repair techniques described 
are microfracture, collagen scaffold enhanced microfracture and different chondrocyte transplantation methods $[13,14]$.

National cohort studies have proven very successful for large-scale investigations of clinical outcome after arthroplasty and ligament reconstruction [15-17]. Data from a national registry potentially represent large amounts of populationbased epidemiological information from multiple centres and surgeons. Therefore, outcome data might be more reliable for a specific surgical intervention. Registry data are potentially more representative for a specific surgical treatment than case series that may contain biased data due to selection of patients and highly dedicated surgeons.

Hip arthroscopy procedures in Denmark have been registered in the Danish Hip Arthroscopy Registry (DHAR) since the beginning of 2012. This registry is a voluntary clinical registry for patients and surgeons, and all of the centres performing hip arthroscopic procedures in Denmark do report to the registry [18]. One of the main indications in the registry for hip arthroscopic surgery is currently femoroacetabular impingement (FAI). The DHAR contains data on cartilage and labral pathology, which enables investigations on both the spectrum of cartilage pathology in FAI patients and the impact of cartilage status on the clinical outcome of arthroscopic FAI management.

The purpose of the present study is to present the intraarticular cartilage pathology in FAI patients and the management strategies to these cartilage lesions based on data from a national cohort. Also we shall investigate the impact of cartilage status on the clinical outcome of arthroscopic FAI management.

\section{Methods}

\section{Clinical registry}

The Danish Hip Arthroscopy Registry (DHAR) was initiated in 2012. Surgeons as well as patients report data online in an ongoing prospective data registration. For this study we extracted pre- and perioperative data including PROMs from DHAR between January 2012 and December 31st 2015. The postoperative patient reported data for this study were extracted at one and two-year follow-up. The registry is approved by the Danish Health Authorities, J.nr. 2012-58-0006.

The patient submits the preoperative subjective scores at registration consisting of various PROM and pain levels. At one, two and five years postoperatively the patient will be notified to submit their PROM scores at follow-up. After surgery, the surgeon reports data from clinical examination, radiological characteristics and the perioperative data.

\section{Patients in the present study}

This study includes patients operated for symptomatic FAI from January 2012 until December 31st 2013, with a minimum of two-year follow-up. DHAR uses the three definitions of FAI types as described by Leunig et al. [3]: cam type, a developed asphericity of the femoral head and widening of the femoral neck, pincer type, a developed global or focal overcoverage on the acetabular side and mixed type, a combination of cam and pincer. We excluded data from patients without cartilage data registration, patients who were diagnosed with other diseases than FAI, previous surgery in the related hip or incomplete data registrations, patients with hip dysplasia defined as centre-edge (CE) angle of less than $25^{\circ}$ and patients with revision hip arthroscopy.

\section{Registry data}

\section{Surgical findings and procedures}

The operative data reported are the surgical procedure times including traction times, labral tears, cartilage injury assessment and surgical technique characteristics such as anchor usage, depth of rim trimming and cam resections.

The definition of acetabular cartilage injury type used in DHAR was initially classified by Beck et al. [2], and was later modified and validated by Konan et al. [19]. We have chosen to use the classification originally described by Beck, which is based on the Outerbridge classification. For femoral cartilage injuries the International Cartilage Repair Society (ICRS)scale is used which is purely based on the injury depth [20].

\section{Patient outcome measurements}

All PROMs used in DHAR are validated self-assessment scores and evaluated as suitable for patients undergoing hip arthroscopy. The used PROM questionnaires are the Copenhagen Hip and Groin Outcome Score (HAGOS), the EQ-5D and the Hip Sports Activity Scale (HSAS). The pain score used is the numeric rating scale (NRS). The HAGOS consists of six subscales assessing symptoms, pain, function in daily living, function in sport and recreation, participation in physical activities and hip and/or groin-related quality of life, each scored separately [21, 22]. HAGOS is a questionnaire (37 questions in total) aimed for young to middle-aged adults undergoing nonsurgical treatment or hip arthroscopy, but also patients presenting with groin pain. The EQ-5D is used as a generic health-related quality of life instrument, which is translated and validated into many languages [23]. The HSAS is recommended as a reliable and valid activity measurement useful for patients with femoroacetabular impingement [24]. Pain levels are measured using the NRS pain scores at rest and after 15 min of walking. A global hip function score using visual analogue scoring (VAS) from 0 to 100 was used, with 100 points being optimal hip function.

\section{Statistical analysis}

The Student $t$-test was used to analyse the differences between the preoperative and postoperative PROM values. $P$-values below 0.05 were considered statistically significant. Subgroup analysis was made between patients with acetabular cartilage injury Beck grade 0-2 and grade 3-4 regarding PROM values using Student's $t$-test for comparisons of PROM values. 
Table 1. Degree of injury for acetabulum and femoral head.

\begin{tabular}{lcc}
\hline & Patients & $\%$ \\
\hline Acetabulum (Becks) & & \\
$\quad$ Grade 0: normal cartilage & 10 & 1.5 \\
Grade 1: fibrillation & 95 & 13.8 \\
Grade 2: wave sign & 281 & 41.0 \\
Grade 3: cleavage tear between labrum & 210 & 30.6 \\
and articular cartilage & & \\
Grade 4: exposed bone in the acetabulum & 90 & 13.1 \\
$\quad$ Cartilage injury $\geq$ Grade 2 & 581 & 84.7 \\
Femoral head (ICRS) & 521 & 75.9 \\
$\quad$ Grade 0: normal cartilage & 52 & 7.6 \\
Grade 1: nearly normal & 68 & 9.9 \\
Grade 2: abnormal & 29 & 4.2 \\
$\quad$ Grade 3: partial loss of cartilage & 16 & 2.3 \\
Grade 4: exposed bone & 113 & 16.5 \\
$\quad$ Cartilage injury $\geq$ Grade 2 & & \\
Combined cartilage injury & 601 & 88.2 \\
$\quad$ Acetabulum $O R$ femoral head cartilage & & \\
injury $\geq$ Grade 2 & 93 & 13.6 \\
$\quad$ Acetabulum $A N D$ femoral head cartilage \\
injury $\geq$ Grade 2
\end{tabular}

\section{Results}

\section{Population characteristics}

Data from a total of 686 FAI procedures in 1082 patients from January 2012 until December 31st 2013 were extracted from DHAR. We excluded 396 procedures due to our exclusion criteria. Forty-seven patients had bilateral procedures performed. Forty-eight percent of the patients were female and $52 \%$ male. The average age at the time of surgery was 38.5 years (range, 12-76 years). The majority of the patients were characterized as mixed type FAI (87.3\%). Isolated pincer type morphology consisted of only $4.9 \%$ whereas $7.8 \%$ were characterized as isolated cam type morphology.

\section{Registration completeness}

The completeness of PROM registrations preoperatively was $58 \%$ and the follow-up completeness at one and two years was $55 \%$ and $52 \%$, respectively. There is an ongoing study that is looking at data completeness comparing data from DHAR to the National Registry for Hospital Procedures.

\section{Cartilage injury findings and surgical procedures}

Cartilage injuries were found in $88 \%$ of cases and the degree of injury for both acetabulum and femoral head is shown in Table 1. Normal cartilage was only found in $1.5 \%$ and $24 \%$ of the acetabular and femoral head joint surfaces, respectively. More severe cartilage injury of Beck or ICRS grade 3-4 was found in 44 and 7\% of the acetabular and femoral head joint surfaces, respectively. Surgery due to cartilage injury was reported in 523 procedures (76\%), with the most commonly performed surgical intervention being
Table 2. Cartilage procedures in patients undergoing arthroscopic FAI surgery.

\begin{tabular}{lcc}
\hline Surgical procedure & Patients & $\%$ \\
\hline Cartilage debridement any location & 560 & 81.6 \\
Cartilage reattachment femoral head & 1 & 0.1 \\
Cartilage reattachment acetabulum & 4 & 0.6 \\
Microfracture femoral head & 3 & 0.4 \\
Microfracture acetabulum & 43 & 6.3 \\
\hline
\end{tabular}

debridement with shaver or radiofrequency ablation technique. (Table 2) Cartilage repair procedure in the form of microfracture was only performed in $7 \%$ of cases. Cartilage flap re-attachment was performed in less than $1 \%$ of cases.

\section{Patient related outcome measures (PROM)}

Overall PROM including pain scores improved significantly from preoperative status to follow-up one and two years postoperatively (Tables 3 and 4). We did not find any significant improvement in these parameters between one and two-year follow-up, except the NRS pain score for walking $(p=0.002)$. HAGOS demonstrated significant improvements in all subscales between the preoperative and the postoperative scores at one-year follow-up.

The impact of having a significant acetabular cartilage injury of Beck grade 3-4 is presented in Tables 3 and 4. We found significantly less improvement of the following PROM parameters when more severe acetabular cartilage injury was present: HAGOS, HSAS and global hip function.

\section{Discussion}

Key findings in the present study are that patients with symptomatic FAI in a large national cohort have significant cartilage changes at the time of surgery, mainly on the acetabular side. Data from the DHAR shows that $88 \%$ of the patients have cartilage changes and $97 \%$ of these were located at the acetabular side. Seventy-seven percent of patients undergo some kind of cartilage procedure during the surgical FAI management.

We also found that patients undergoing FAI surgery do benefit from hip arthroscopic procedures involving labral reinsertion, cartilage surgery and removal of cam and pincer bone deformities within a 2-year follow-up. We also found that patients with cartilage injury grade 3 to 4 have inferior results than those with no or minor cartilage injury.

A systematic review by Nwachukwu et al. compared open vs. arthroscopic FAI surgery and demonstrated excellent and comparable hip survival rates at medium to long-term follow-up. Hip arthroscopy showed a statistically significant improvement in general health-related quality of life, when compared to open procedures [25]. In the study with THA as an outcome endpoint, there was an overall survival rate of $93 \%$ for open and $90.5 \%$ for arthroscopic procedures. A recent retrospective population-based analysis $(n=7351)$ by Schairer et al. showed an overall conversion rate of $12.4 \%$ within two 
Table 3. Influence of cartilage injury on patient reported outcome in patients undergoing arthroscopic FAI surgery.

\begin{tabular}{|c|c|c|c|c|c|c|c|c|}
\hline & \multicolumn{4}{|c|}{1 year data } & \multicolumn{4}{|c|}{2 year data } \\
\hline & \multicolumn{2}{|c|}{ Grade $0-2$} & \multicolumn{2}{|c|}{ Grade 3-4 } & \multicolumn{2}{|c|}{ Grade $0-2$} & \multicolumn{2}{|c|}{ Grade 3-4 } \\
\hline & Mean & $S D$ & Mean & $S D$ & Mean & $S D$ & Mean & $S D$ \\
\hline \multicolumn{9}{|l|}{ HAGOS } \\
\hline Pain & 73 & 23 & 70 & 21 & 74 & 22 & 72 & 20 \\
\hline Symptoms & 68 & 21 & 66 & 19 & 69 & 21 & 68 & 19 \\
\hline ADL & 75 & 24 & 73 & 24 & 77 & 25 & 74 & 23 \\
\hline Sport & 61 & 30 & 56 & 27 & 64 & 29 & $55^{*}$ & 28 \\
\hline $\mathrm{PA}$ & 48 & 35 & $39^{*}$ & 34 & 51 & 35 & $40^{*}$ & 36 \\
\hline QOL & 55 & 27 & 50 & 26 & 59 & 26 & $51^{*}$ & 26 \\
\hline NRS - rest & 19 & 22 & 19 & 21 & 19 & 24 & 16 & 18 \\
\hline NRS - activity & 28 & 31 & 30 & 28 & 23 & 25 & 21 & 22 \\
\hline VAS & 29 & 25 & 33 & 23 & 28 & 25 & $34 *$ & 25 \\
\hline EQ5D & 0.78 & 0.19 & 0.76 & 0.17 & 0.79 & 0.19 & 0.77 & 0.16 \\
\hline HSAS & 3.1 & 2.1 & 3.2 & 2.0 & 3.3 & 2.0 & 3.3 & 1.9 \\
\hline
\end{tabular}

* Significant difference between grade $0-2$ and grade $3-4, p<0.05$.

Table 4. Outcome differences between 2 years FU and baseline values.

\begin{tabular}{lccccc}
\hline & \multicolumn{4}{c}{ 2 year data } \\
\cline { 2 - 3 } \cline { 5 - 6 } \cline { 5 - 6 } & \multicolumn{2}{c}{ Grade 0-2 } & & Grade 3-4 \\
\cline { 2 - 3 } & Mean & $S D$ & & & Mean \\
\hline HAGOS & 22 & 20 & & 17 & 21 \\
$\quad$ Pain & 19 & 19 & & 15 & 21 \\
Symptoms & 25 & 23 & & 19 & 25 \\
ADL & 29 & 27 & & $18^{*}$ & 30 \\
Sport & 33 & 34 & & $16^{*}$ & 40 \\
PA & 29 & 25 & & $17^{*}$ & 23 \\
QOL & 20 & 28 & & 18 & 26 \\
NRS - rest & 30 & 40 & & 25 & 30 \\
NRS - activity & 29 & 26 & & $17^{*}$ & 30 \\
VAS & 0.14 & 0.18 & & 0.20 & 0.20 \\
EQ5D & 0.9 & 2.2 & & $0.4^{*}$ & 1.8 \\
HSAS & & & &
\end{tabular}

* Significant difference between grade $0-2$ and grade $3-4$, $p<0.05$.

years of hip arthroscopic surgery [26]. The lowest rate of conversion was found in the age group younger than 40 years, at $3 \%$. The highest risk of conversion was seen in the age group 60 to 69 years, at $35 \%$.

A systematic review by Weber et al. described a rate of reoperations after hip arthroscopy at $6.3 \%$ in a cohort consisting of more than 6000 from 92 studies [27]. The majority of these reoperations were, respectively, revision hip arthroscopy in $1.9 \%$ and conversion to THA in $2.9 \%$ of the patients.

In a recently published study by Malviya et al. of more than 6000 hip arthroscopies registered between 2005 and 2013 in the British National Health Service, a revision hip arthroscopy rate of $4.5 \%$ was found [28]. Their conversion rate to THR was $10.6 \%$. In the present study, revision hip arthroscopy was performed in $7.3 \%$ of cases and conversion to THA was reported in $1.6 \%$ of the cases.

We found that a large proportion $(88 \%)$ of this FAI cohort had chondral injuries at the time of surgery. The most common site of cartilage injury was in the acetabulum and to a minor degree on the femoral head. Although many of these injuries were described as full thickness cartilage damage, a repair procedure was not frequently performed. In the DHAR, debridement and radiofrequency ablation of cartilage injuries were the most common procedures compromising more than $91 \%$ of all cartilage procedures.

In a large cross-sectional study of a North American FAI cohort, the most common surgical technique during FAI surgery was femoral head-neck osteochondroplasty in $91.6 \%$ of the 1130 procedures. However, CAM deformity resection was not considered a cartilage procedure in the DHAR. The percentage of acetabular microfracture interventions in this study was similar to the reported number in DHAR, respectively, 5.6\% and 5.4\% [29].

Several cartilage repair strategies have been suggested for management of the cartilage lesion found during hip arthroscopy in FAI patients. Early changes can be managed by the debridement of bulging and loose cartilage flaps. More advanced methods are suturing and gluing of cartilage flaps to different repair techniques for areas of cartilage loss [10-12]. The repair techniques described are microfracture, collagen scaffold enhanced microfracture and different chondrocyte transplantation methods $[13,30,31]$. Microfracture as described by Steadman et al. [32] is a readily available cartilage repair technique for hip arthroscopy. The efficacy for hip cartilage repair has still not been investigated. But experience from the knee has shown that microfracture can form fibrocartilage that can result in symptom relief in $70 \%$ of patients [33]. One single case series of 24 FAI patients with microfracture treatment of cartilage lesions and second-look arthroscopic evaluation of healing found excellent filling in 95\% patients. Histologic evaluation of repair tissue from two patients demonstrated fibrocartilage tissue characteristics [34].

Microfracture repair can be enhanced with the application of a collagen scaffold on top of the defect after microfracture using the so-called autologous matrix-induced chondrogenesis (AMIC) technique. This technique was compared with chondrocyte transplantation by Mancini and Fontana describing good outcomes long-term with both techniques resulting in 
38-39 point improvements in modified Harris hip score without any difference between techniques [13].

One major problem for investigations of the impact of cartilage procedures in FAI surgical management is that the cartilage procedure is secondary to procedures done to remove the FAI pathology such as removal of cam and pincer deformities and labral repairs. A recent systematic review on cartilage injury management by Marquez-Lara et al states that only level IV and V studies are present and that there is a need for good level I studies [14].

The present study and studies previously mentioned show consistently improved clinical outcomes of hip arthroscopy in the treatment of FAI and the concurrent labral damage, especially regarding patient's quality of life and pain-related outcome measures. However, the impact of cartilage debridement and repair is poorly investigated and there is a major challenge scientifically since the cartilage procedures are secondary to the FAI procedures. There is a need for good level I studies comparing different surgical treatment strategies of FAI with and without cartilage management.

\section{Limitations}

There are limitations in the present study with potential data quality issues due to the potential diversity in interpretation of the intraarticular pathology findings done by the individual surgeons and due to completeness of data in the Danish Hip Arthroscopy Registry. All data input is voluntary, both for surgeons and patients. We know from the Danish National ACL Registry that patient inputs are as low as 35\% but more than $85 \%$ from surgeons [35]. The hip arthroscopy procedure is regulated by the Danish Board of Health and is therefore limited to the 11 centres with permission to perform the procedure. The actual number of hip arthroscopic procedures during this time period in Denmark is not known, but is being studied in an ongoing validation study. Further studies based on central healthcare registries are needed to document the degree of completeness for surgical data. Finally, DHAR does not include data from patients undergoing nonsurgical treatments of FAI, which would be of interest for outcome studies. However, the DHAR utilizes prospective data collection without potential bias from future study purposes such as the present study. The high data volume and the diversity of clinics and surgeons potentially provide data that to a high degree reflects the true pathology status and outcome profile of FAI patients.

\section{Conclusion}

The majority of patients with femoroacetabular impingement undergoing hip arthroscopy have significant cartilage changes at the time of surgery primarily at the acetabulum and to a lesser degree at the femoral head. During FAI surgery the majority of patients have cartilage debridement performed but rarely cartilage repair. The presence of severe cartilage injury at the time of arthroscopic FAI surgery results in reduced subjective outcome and hip function.

\section{Conflict of interest}

The authors declare no conflict of interest in relation with this paper.

\section{References}

1. Ganz R et al. (2003) Femoroacetabular impingement: a cause for osteoarthritis of the hip. Clin Orthop Relat Res 417, 112-120.

2. Beck M, Kalhor M, Leunig M, Ganz R (2005) Hip morphology influences the pattern of damage to the acetabular cartilage: femoroacetabular impingement as a cause of early osteoarthritis of the hip. J Bone Joint Surg Br 87(7), 1012-1018.

3. Leunig M, Beck M, Dora C, Ganz R (2006) Femoroacetabular impingement: trigger for the development of coxarthrosis. Orthopade 35(1), 77-84.

4. Ganz R, Leunig M, Leunig-Ganz K, Harris WH (2008) The etiology of osteoarthritis of the hip: an integrated mechanical concept. Clin Orthop Relat Res 466(2), 264-272.

5. Nicholls AS et al. (2011) The association between hip morphology parameters and nineteen-year risk of end-stage osteoarthritis of the hip: a nested case-control study. Arthritis Rheum 63(11), 3392-3400.

6. Gosvig KK, Jacobsen S, Sonne-Holm S, Palm H, Troelsen A (2010) Prevalence of malformations of the hip joint and their relationship to sex, groin pain, and risk of osteoarthritis: a population-based survey. J Bone Joint Surg Am 92(5), $1162-1169$.

7. Hetaimish BM, et al. (2013) Consistency of reported outcomes after arthroscopic management of femoroacetabular impingement. Arthroscopy 29(4), 780-787.

8. Siebenrock KA, Fiechter R, Tannast M, Mamisch TC, von Rechenberg B (2013) Experimentally induced cam impingement in the sheep hip. J Orthop Res 31(4), 580-587.

9. Fontana A, Mancini D, Gironi A, Acerbi A (2016) Hip osteochondral lesions: arthroscopic evaluation. Hip Int 26(Suppl 1), 17-22.

10. Stafford GH, Bunn JR, Villar RN (2011) Arthroscopic repair of delaminated acetabular articular cartilage using fibrin adhesive Results at one to three years. Hip Int 21(6), 744-750

11. Cassar-Gheiti AJ et al. (2015) Comparison of four chondral repair techniques in the hip joint: a biomechanical study using a physiological human cadaveric model. Osteoarthritis Cartilage 23(6), 1018-1025.

12. Kaya M, Hirose T, Yamashita T (2015) Bridging suture repair for acetabular chondral carpet delamination. Arthrosc Tech 4(4), e345-e348.

13. Mancini D, Fontana A (2014) Five-year results of arthroscopic techniques for the treatment of acetabular chondral lesions in femoroacetabular impingement. Int Orthop 38(10), 2057-2064.

14. Marquez-Lara A et al. (2016) Arthroscopic management of hip chondral defects: a systematic review of the literature. Arthrosc J Arthrosc Relat Surg 32(7), 1435-1443.

15. Havelin LI et al. (2000) The Norwegian Arthroplasty Register: 11 years and 73,000 arthroplasties. Acta Orthop Scand 71(4), 337-353.

16. Granan L-P, Forssblad M, Lind M, Engebretsen L (2009) The Scandinavian ACL registries 2004-2007: baseline epidemiology. Acta Orthop 80(5), 563-567. 
17. Lind M et al. (2012) Medium to long-term follow-up after ACL revision. Knee Surg Sports Traumatol Arthrosc 20(1), 166-172.

18. Mygind-Klavsen B et al. (2016) Danish Hip Arthroscopy Registry: an epidemiologic and perioperative description of the first 2000 procedures. J Hip Preserv Surg 3, 138-145.

19. Konan S, Rayan F, Meermans G, Witt J, Haddad FS (2011) Validation of the classification system for acetabular chondral lesions identified at arthroscopy in patients with femoroacetabular impingement. J Bone Joint Surg Br 93(3), $332-336$

20. van den Borne MPJ et al. (2007) International Cartilage Repair Society (ICRS) and Oswestry macroscopic cartilage evaluation scores validated for use in Autologous Chondrocyte Implantation (ACI) and microfracture. Osteoarthritis Cartilage 15(12), 1397-1402.

21. Thorborg K, Hölmich P, Christensen R, Petersen J, Roos EM (2011) The Copenhagen Hip and Groin Outcome Score (HAGOS): development and validation according to the COSMIN checklist. Br J Sports Med 45(4), 478-491.

22. Thorborg $\mathrm{K}$ et al. (2011) Patient-Reported Outcome (PRO) questionnaires for young to middle-aged adults with hip and groin disability: a systematic review of the clinimetric evidence. Br J Sport Med 45(6), 478-491.

23. Zampelis V, Ornstein E, Franzén H, Atroshi I (2014) A simple visual analog scale for pain is as responsive as the WOMAC, the SF-36, and the EQ-5D in measuring outcomes of revision hip arthroplasty. Acta Orthop 85(2), 128-132.

24. Naal FD et al. (2013) The Hip Sports Activity Scale (HSAS) for patients with femoroacetabular impingement. Hip Int 23(2), 204-211.

25. Nwachukwu BU et al. (2016) Arthroscopic versus open treatment of femoroacetabular impingement: a systematic review of medium- to long-term outcomes. Am J Sports Med 44(4), 1062-1068.

26. Schairer WW, Nwachukwu BU, McCormick F, Lyman S, Mayman D (2016) Use of hip arthroscopy and risk of conversion to total hip arthroplasty: a population-based analysis. Arthroscopy 32(4), 587-593.

27. Weber AE, Harris JD, Nho SJ (2015) Complications in Hip Arthroscopy: A Systematic Review and Strategies for Prevention. Sports Med Arthrosc 23(4), 187-193.

28. Malviya A et al. (2015) Complications and survival analyses of hip arthroscopies performed in the national health service in england: a review of 6,395 Cases. Arthroscopy 31(5), 836-842.

29. Clohisy JC et al. (2013) Descriptive epidemiology of femoroacetabular impingement: a North American cohort of patients undergoing surgery. Am J Sport Med 41(6), $1348-1356$.

30. Körsmeier $\mathrm{K}$ et al. (2016) Arthroscopic three-dimensional autologous chondrocyte transplantation using spheroids for the treatment of full-thickness cartilage defects of the hip joint. Knee Surgery, Sport Traumatol Arthrosc 24(6), 2032-2037.

31. Fickert S, Schattenberg T, Niks M, Weiss C, Thier S (2014) Feasibility of arthroscopic 3-dimensional, purely autologous chondrocyte transplantation for chondral defects of the hip: a case series. Arch Orthop Trauma Surg 134(7), 971-978.

32. Steadman JR, Rodkey WG, Rodrigo JJ (2001) Microfracture: surgical technique and rehabilitation to treat chondral defects. Clin Orthop Relat Res (391 Suppl), S362-S369.

33. Mithöfer K, Minas T, Peterson L, Yeon H, Micheli LJ (2005) Functional outcome of knee articular cartilage repair in adolescent athletes. Am J Sports Med 33(8), 1147-1153.

34. Karthikeyan S, Roberts S, Griffin D (2012) Microfracture for acetabular chondral defects in patients with femoroacetabular impingement: results at second-look arthroscopic surgery. Am J Sports Med 40(12), 2725-2730.

35. Lind M, Menhert F, Pedersen AB (2009) The first results from the Danish ACL reconstruction registry: epidemiologic and 2 year follow-up results from 5,818 knee ligament reconstructions. Knee Surgery, Sport Traumatol Arthrosc 17(2), 117-124.

Cite this article as: Lund B, Nielsen TG \& Lind M (2017) Cartilage status in FAI patients - results from the Danish Hip Arthroscopy Registry (DHAR). SICOT J, 3, 44 